\title{
The protective role and mechanism of liriodendrin in rats with myocardial infarction
}

\author{
Bo Li1 $^{1 \#}$, Bo-Chen Yao ${ }^{2 \#}$, Qing-Liang Chen ${ }^{2}$, Yan-Qiu Song ${ }^{3}$, Nan Jiang $^{2}$, Li-Li Zhao ${ }^{3}$, Zhi-Gang Guo ${ }^{2}$ \\ ${ }^{1}$ Clinical School of Thoracic, Tianjin Medical University, Tianjin, China; ${ }^{2}$ Department of Cardiovascular Surgery, Tianjin Chest Hospital, Tianjin, \\ China; ${ }^{3}$ Tianjin Institute of Cardiovascular Diseases, Tianjin, China \\ Contributions: (I) Conception and design: B Li, BC Yao, ZG Guo; (II) Administrative support: ZG Guo, QL Chen, N Jiang; (III) Provision of study \\ materials or patients: B Li, BC Yao, QL Chen, YQ Song, N Jiang, LL Zhao; (IV) Collection and assembly of data: B Li, BC Yao, QL Chen; (V) Data \\ analysis and interpretation: QL Chen, N Jiang, ZG Guo; (VI) Manuscript writing: All authors; (VII) Final approval of manuscript: All authors. \\ "These authors contributed equally to this work. \\ Correspondence to: Zhi-Gang Guo. Department of Cardiovascular Surgery, Tianjin Chest Hospital, 261 Taierzhuang Road, Tianjin 300001, China. \\ Email: 314067280@qq.com.
}

Background: Liriodendrin is a therapeutic constituent of sargentgloryvine stem which is a famous Chinese traditional medicine. Previous studies have suggested liriodendrin could inhibit different pathways to treat inflammation in lung and intestinal tract. But whether it can treat myocardial infarction (MI) is unknown. We investigated the protective effect of liriodendrin on acute MI in rats and explored the specific mechanisms to expand the use of this traditional Chinese medicine.

Methods: The rats were randomized into the sham group (sham operation), control group (ligation of the left anterior descending artery), and liriodendrin group. The liriodendrin group was intragastrically administered with a liriodendrin solution $(100 \mathrm{mg} / \mathrm{kg})$. The control group and the sham group were intragastrically administered with normal saline. Before all rats were euthanized, echocardiography was used to detect their cardiac function. Hematoxylin and eosin (HE) staining and the terminal deoxynucleotidyl transferase-mediated nick end labeling (TUNEL) method were performed. Further quantitative detection of interleukin-1 $\beta$ (IL-1 $\beta$ ) and tumor necrosis factor- $\alpha(\mathrm{TNF}-\alpha)$ levels in tissues were also detected. Western Blot and real-time polymerase chain reaction (RT-PCR) were used to detect the apoptosis and nuclear factor kappa-B (NF-кB) pathway in tissues. H9C2 cells were used to detect the related mechanisms in vitro.

Results: Echocardiography showed that, compared to control group, the cardiac function of the liriodendrin group was significantly improved. histopathological staining of the control group showed that the myocardial tissue was severely damaged, and inflammatory cells were infiltrated. Compared to the control group, the apoptosis index of the liriodendrin group was significantly lower $(\mathrm{P}<0.05)$. Enzyme-linked immunosorbent assay (ELISA) results showed that the levels of IL- $1 \beta$ and TNF- $\alpha$ in the control group were higher than those in the liriodendrin group $(\mathrm{P}<0.05)$. Meanwhile, apoptosis and the NF- $\kappa \mathrm{B}$ pathway were inhibited after liriodendrin administration $(\mathrm{P}<0.05)$. Moreover, the mRNA transcriptional activity in the control group was also higher than that in the liriodendrin group $(\mathrm{P}<0.05)$. Because of the effect of liriodendrin, $\mathrm{NF}-\kappa \mathrm{B}$ pathway and apoptosis were downregulated in $\mathrm{H} 9 \mathrm{C} 2$ cells which were exposed to ischemia-hypoxia.

Conclusions: Liriodendrin may protect myocardial cells after myocardial infarction in rats by inhibiting the release of inflammatory factors, activation of the NF- $\kappa$ B pathway, and apoptosis.

Keywords: Coronary heart disease; myocardial infarction (MI); liriodendrin; apoptosis; NF-кB pathway

Submitted Nov 15, 2021. Accepted for publication Jan 17, 2022.

doi: $10.21037 /$ jtd-21-1998

View this article at: https://dx.doi.org/10.21037/jtd-21-1998 


\section{Introduction}

Myocardial infarction (MI) is a serious disease of cardiovascular disease and is the main cause of cardiovascular disease morbidity and mortality in the world (1). After MI occurs, the innate immune response is activated, different kinds of inflammatory cells infiltrate damaged tissue, and a mass of cytokines, such as tumor necrosis factor- $\alpha(\mathrm{TNF}-\alpha)$ and interleukin-6 (IL-6), are produced. The number of cardiomyocyte apoptosis increases, myocardial collagen fibers increase, the heart undergoes pathological remodeling, and the cardiac function is significantly reduced (2). Nuclear factor kappa B $(\mathrm{NF}-\mathrm{\kappa B})$ also plays a crucial part in the pathogenesis of MI.

A rich variety of traditional Chinese medicines are safe to eat and have received widespread attention in recent years. A large number of studies have shown that monomers found in traditional Chinese medicine can inhibit the inflammatory reaction and reduce the expression and secretion of IL$1 \beta$, interleukin-6 (IL-6), and other inflammatory factors in animal models of myocardial infarction. For example, the combination of tanshinone IIA and puerarin (1:1) can observably reduce the production and secretion of IL$1 \beta$, IL- 6 , and other inflammatory factors in the early stage after myocardial infarction (3). Research has also shown that tripterygium wilfordii extract can inhibit the receptor for advanced glycation end-products/NF- $\kappa \mathrm{B}$ (RAGE/NF$\kappa \mathrm{B})$ pathway and improve cardiac function. Furthermore, sargentodoxa cuneata is a famous medicine in China and can cure abdominal pain caused by acute appendicitis, ulcers, and traumatic swelling. Liriodendrin is one of the various chemical components of sargentodoxa cuneata and possesses a variety of biological functions, including preventing tumors, inflammation, oxidation, and platelet aggregation. Previous studies have demonstrated that these effects of liriodendrin occur because liriodendrin can down-regulate the release of various inflammatory factors $(4,5)$. However, the effects above of liriodendrin were mainly reported in the domain of abdominal and lung inflammation. Previous studies have shown that inflammation due to the necrosis of myocardial cells after $\mathrm{MI}$ is the main cause of further myocardial damage and ventricular remodeling. The product of cell necrosis can attract a large number of inflammatory cells, such as macrophages, neutrophils, etc., to the lesion area. These inflammatory cells can release a large number of inflammatory factors, further expand the inflammatory effect in the lesion area, and activate multiple pathways, further promote myocardial apoptosis, leading to ventricular remodeling. Whether liriodendrin exerts a protective effect during MI, either in animals or humans, has not been reported. Therefore, based on our rat MI model, we studied the effect of liriodendrin on improving ventricular remodeling and its possible mechanism, especially the relationship between liriodendrin, improved cardiac function, and anti-apoptosis. We present the following article in accordance with the ARRIVE reporting checklist (available at https://jtd.amegroups.com/article/ view/10.21037/jtd-21-1998/rc).

\section{Methods}

\section{Experimental animals}

A protocol was prepared before the study without registration. Wistar rats were purchased from Beijing Huafukang Experimental Animal Technology Co. (Beijing, China), and all rodents were bred in the animal housing facility of the Institute of Cardiovascular Diseases. The study was approved by the Animal Ethics Committee of Tianjin Chest Hospital (No. TJCH-2021-007), and performed according to the guidelines of the Chinese Ethics Committee for the care and use of animals (6).

\section{Drug and reagents}

Liriodendrin was extracted from Tianjin Nankai Hospital, and its purity was $>97 \%$. AnaeroPacks were purchased from Mitsubishi Gas Chemical Company (Tokyo, Japan). ELISA kits for detecting rats IL- $1 \beta$ and TNF- $\alpha$ were supplied by R\&D Systems (Minneapolis, MN, USA). TUNEL kits for detecting myocardial cell apoptosis were supplied by Bioss Antibodies (Beijing, China). All western blot antibodies were supplied by Cell Signaling technology (Danvers, MA, USA). Bicinchoninic Acid Assay reagent kits were purchased from Thermo Scientific (Waltham, MA, USA). The RTPCR kit was purchased from R\&D Systems (Minneapolis, $\mathrm{MN}, \mathrm{USA})$.

\section{Cell culture}

H9c2 Cardiomyocytes were cultured in dulbecco's modified eagle's medium (DMEM) medium containing $10 \%$ fetal bovine serum (FBS), $100 \mathrm{U} / \mathrm{mL}$ of streptomycin, and $100 \mathrm{U} / \mathrm{mL}$ of penicillin in a $37{ }^{\circ} \mathrm{C}, 5 \% \mathrm{CO}_{2}$ cell incubator. The cells were seeded in a 6 -well plate, and different concentrations of liriodendrin were 
pretreated for $24 \mathrm{~h}$ prior to ischemia-hypoxia exposure. The original medium was replaced with serum-free medium after $24 \mathrm{~h}$ and different concentrations of liriodendrin were added into the medium again. Then 6-well plate was placed in a sealable jar with AnaeroPacks for $24 \mathrm{~h}$. Presence of $\mathrm{O}_{2}$ can be checked by the color change of the oxygen indicator in the jar

\section{MI modeling and grouping}

All the rats, which were male and weighed $200 \pm 10$ g, were randomized into three groups: the sham, model, and liriodendrin groups $(\mathrm{n}=18)$. After anesthetization (pentobarbital sodium $50 \mathrm{mg} / \mathrm{kg}$, intraperitoneally) and intubation, a left thoracotomy was performed, and the left anterior descending artery (LAD) ligation was visualized. LAD was encircled with a 6-0 Prolene and permanently ligated. We did not apply this ligation process to the sham group. After myocardial ischemia was visually observed, the chest was closed with three layers of sutures. The liriodendrin group received liriodendrin $(100 \mathrm{mg} / \mathrm{kg})$ by intragastric administration from 5 days before the LAD ligation operation to 5 days after the operation, while the sham and control groups were administered distilled water. Five days after the operation, all rats were euthanized.

\section{Echocardiography}

Echocardiograms were obtained to measure the left ventricular end-systolic diameter (LVIDs), left ventricular end-diastolic diameter (LVIDd), left ventricular ejection fraction (EF), left ventricular end-diastolic volume (EDV), and left ventricular end-systolic volume (ESV) in rats prior to euthanization.

\section{Histopathological staining}

Hearts of the three groups were harvested for routine histopathological analysis. After the hearts were formalinfixed, paraffin-embedded tissues were cut at $4 \mu \mathrm{m}$ thickness and stained with hematoxylin and eosin (HE). Three hearts were taken from each group to performed terminal deoxynucleotidyl transferase-mediated nick end labeling (TUNEL). Four slices of each heart were observed, and 5 fields were randomly selected in each slice $(\times 200)$. We calculated the percentage of TUNEL positive cells relative to the total number of cells in each field and took the average value as the apoptotic index (AI).

\section{Enzyme-linked immunosorbent assay (ELISA) analysis}

Left ventricular myocardial tissue was harvested and sonicated in phosphate buffered saline (PBS). IL-1 $\beta$ and TNF- $\alpha$ levels in the supernatant, which was obtained from the homogenate after centrifugation, were measured using an ELISA kit. Assays were performed following the manufacturer's instructions. All cell supernatant was also collected to perform the ELISA test.

\section{Western blot analysis}

Ice-cold radio-immunoprecipitation assay (RIPA) lysis buffer was used to incubate the hearts of the rats and cells. Protein was analyzed using 10\% SDS-polyacrylamide gel electrophoresis, then the protein was transferred to polyvinylidene fluoride (PVDF) membranes. The membrane was washed with phosphate-buffered saline after it was blocked in Tris-buffered saline (TBS). The blot was probed using an enhanced chemiluminescence kit. Antibodies against B-cell lymphoma-2 (Bcl-2), Bcl-2 associated $\mathrm{X}$ (Bax), cleaved caspase- 3 , IкB $\alpha, \mathrm{NF}-\kappa \mathrm{B}, \mathrm{IKK}-\mathrm{b}$, $\mathrm{p}-\mathrm{NF}-\kappa \mathrm{B}$, and $\mathrm{p}-\mathrm{I} \kappa \mathrm{B} \alpha$ were used in the western blot assay to detect apoptosis and NF- $\mathrm{KB}$ pathway activation.

\section{Transcriptional activity of anti-apoptotic protein ribonucleic acid (RNA)}

Total RNA of H9c2 cells and myocardial tissue was extracted by Trizol. The RNA was then reverse transcribed into cDNA using TransScript Allin-One First-Strand cDNA Synthesis SuperMix for qPCR. Amplified cDNA was analyzed using real-time PCR (BIO-RAD) with the following primers: Bcl-2: F5'-CTTTGAGTTCGGTGGGGTCA-3', R5'-AGTTCCACAAAGGCATCCCAG-3'; Cleaved caspase-3: F5'-AGCACTGGAATGTCAGCTCGC-3', R5 '-CAGGTCCACAGGTCCGTTCG-3'; Bax: F5 ' - ACCAAGAAGCTGAGCGAGTG-3', R5 ' TCCACATCAGCAATCATCCTCT-3'; and GAPDH: F5'-ACAAAGTGGACATTGTTGCC-3', GAPDH: R5'CTCCATGGTGGTGAAGACGC-3'.

\section{Statistical analysis}

The data was statistically analyzed using SPSS 23.0b (IBM Corp., Armonk, NY, USA) and GraphPad Prism (version 5.0, GraphPad Software, San Diego, CA, USA). Differences among multiple groups were compared by using a one-way 


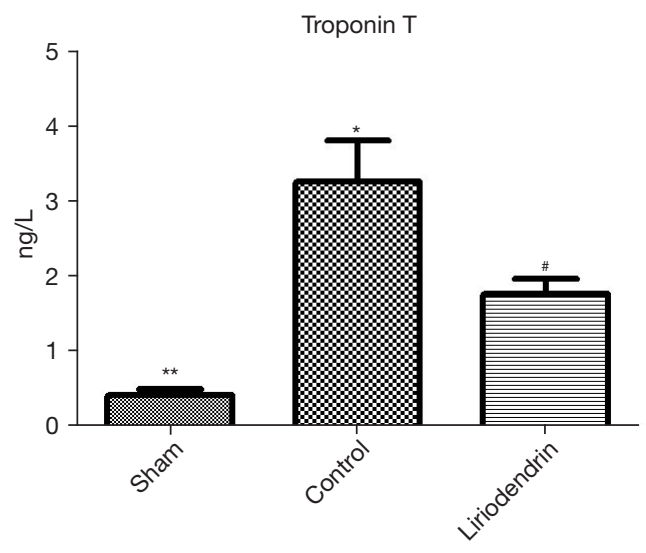

Figure 1 The value of troponin $\mathrm{T}$ was the highest in the control group, compared to the other two groups. The value of troponin $\mathrm{T}$ decreased significantly in the liriodendrin group. *: control group vs. sham group, $\mathrm{P}<0.05$; ": control group vs. liriodendrin group, $\mathrm{P}<0.05$; ** liriodendrin group vs. sham group, $\mathrm{P}<0.05$.

analysis of variance (ANOVA) with a post-hoc NewmanKeuls test. Differences were considered statistically significant at $\mathrm{P}<0.05$.

\section{Results}

\section{Liriodendrin reduces myocardial injury in myocardial infarction rats}

In order to evaluate the quality level of the rat MI model and the therapy effect of liriodendrin on MI, troponin T, a specific marker of myocardial injury, was detected in all three groups. Two hours after the operation, the level of troponin $\mathrm{T}$ in the control group was significantly higher than that in the sham group $(\mathrm{P}<0.05)$. Compared with the control group, the troponin $\mathrm{T}$ level in the liriodendrin group was remarkable lower $(\mathrm{P}<0.05)$ (Figure 1).

\section{Liriodendrin improves left ventricular remodeling in rats with MI}

To further investigate the potential effect of liriodendrin in clinical treatment, we tested if cardiac function can be improved through oral administration. In the control group, part of the anterior wall of the left ventricle was thinned, and the myocardial structure of the anterior wall and anteroseptal disappeared. Reduction of left ventricular ejection fraction (LVEF) in the liriodendrin group was minimum compared with the control group and the sham group, which indicated that the treatment of liriodendrin led to significant changes in LVEF. LVIDs, LVIDd, EDV, and ESV were lower in the liriodendrin group, and the differences were statistically significant $(\mathrm{P}<0.05)$, which demonstrated that liriodendrin improved left ventricular remodeling in rats with MI. There were no significant differences in LVIDs, LVIDd, EDV, and ESV between the sham group and the liriodendrin group $(\mathrm{P}>0.05)$ (Figure 2).

\section{Liriodendrin reduced the MI size and inbibited inflammatory cell infiltration in rats}

HE staining of the myocardium in the control group showed that the myocardial tissue structure disappeared and the morphology of myocardial cells was not clear. Karyopyknosis and karorrhexis were notably observed. We also observed a large number of inflammatory cells that infiltrated the myocardium. The myocardial necrosis in the liriodendrin group was significantly lighter than that of the control group, including partial disappearance of the myocardial tissue structure, slight dilation of small blood vessels in the myocardium, and reduction of inflammatory cells infiltration. In the sham group, myocardial cells were arranged in an orderly manner and myocardial tissue structure was clear (Figure 3).

\section{Liriodendrin inhibited myocardial apoptosis in rats with MI}

As shown in Figure 4, the terminal deoxynucleotidyl TUNEL technique showed that the number of apoptotic cells (TUNEL positive) in the MI area in the control group was significantly increased $(\mathrm{P}<0.05)$ as compared with the control group. After treatment with liriodendrin, the apoptosis index of myocardial cells decreased significantly, and the difference between the model and liriodendrin group was statistically significant $(\mathrm{P}<0.01)$ (Figure 4).

\section{Liviodendrin inbibited inflammatory response in rats with MI}

As a key proinflammatory cytokine, IL-1 $\beta$ is involved in a variety of autoimmune inflammatory responses and cellular activities. IL- $1 \beta$ can regulate apoptosis and promote IL- 6 and TNF- $\alpha$ synthesis. TNF- $\alpha$, which is the initial factor of the NF- $\mathrm{KB}$ pathway, is also an important inflammatory factor. The levels of IL-1 $\beta$ and TNF- $\alpha$ in myocardial tissue of the control group were notably higher $(\mathrm{P}<0.05)$ in comparison with the sham and liriodendrin 

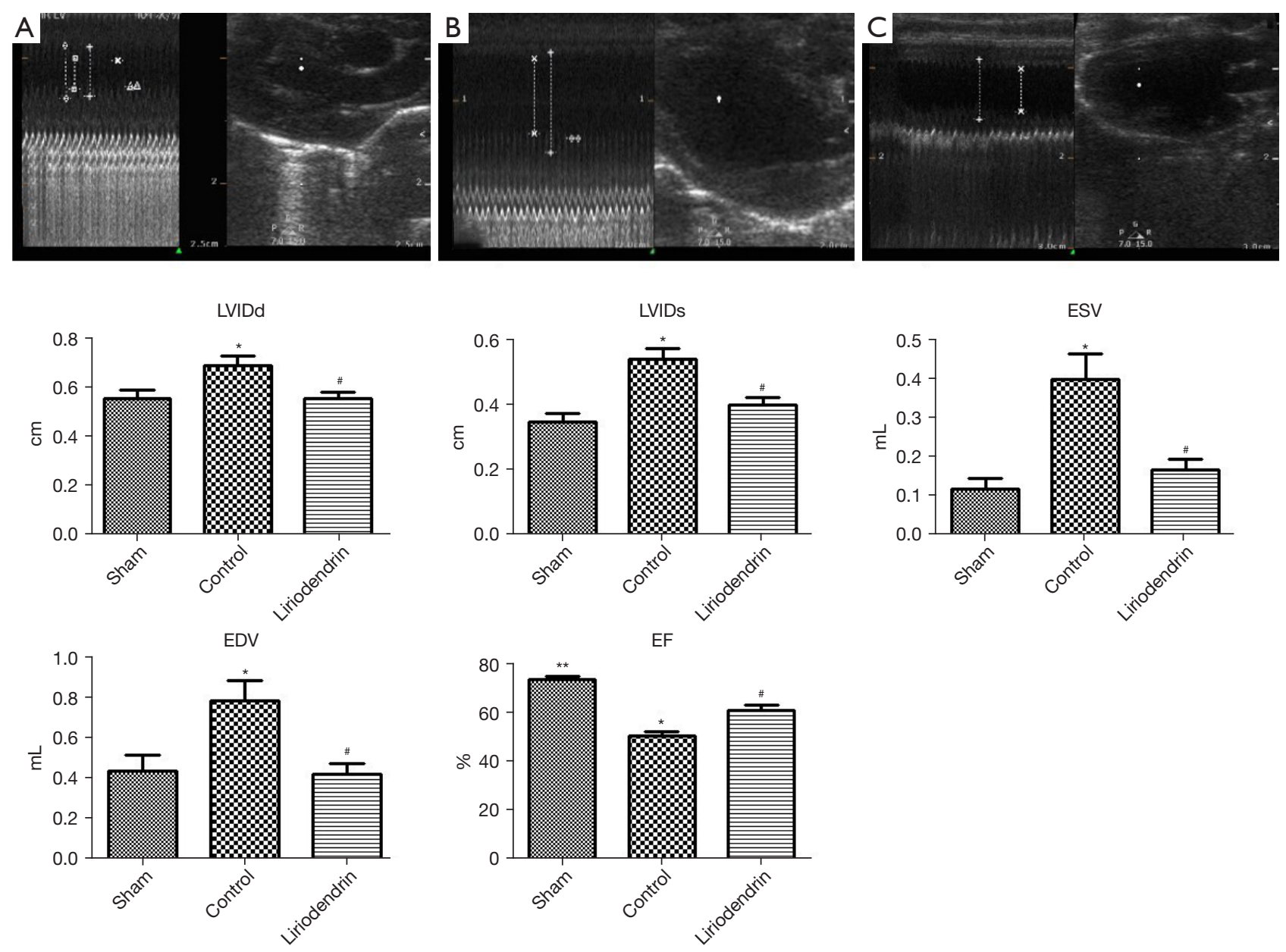

Figure 2 Parameters of cardiac function obtained from echocardiographic images (mean \pm SEM). As shown in the figure, EF of the control group decreased significatively and decreased the least in the liriodendrin group. Compared to the control group, EDV, ESV, LVIDd, and LVIDs of the liriodendrin group expanded less significantly. (A) Sham group; (B) control group; and (C) liriodendrin group. *: control group vs. sham group; $\mathrm{P}<0.05$. * : control group vs. liriodendrin group, $\mathrm{P}<0.05$; **: liriodendrin group vs. sham group, $\mathrm{P}<0.05$. LVIDd, left ventricular end-diastolic diameter; LVIDs, left ventricular end-systolic diameter; ESV, end-systolic volume; EDV, end-diastolic volume; EF, ejection fraction; SEM, standard error of the mean.

groups (Figure 5). The levels of inflammatory mediators in the liriodendrin group were decreased compared with the control group, which suggested that liriodendrin could effectively inhibit the release of inflammatory factors and prevent activation of the inflammatory response.

\section{Liriodendrin down-regulates the expression of apoptosis proteins in rats with MI}

Bcl-2 is derived from the Bcl-2 gene family and has a significant anti-apoptosis effect. Bax, which is also derived from the Bcl-2 gene family, has a notable apoptosispromoting effect which is opposite to Bcl-2. Cleaved caspase- 3 is an activated form of caspase- 3 , which is the executor of apoptosis and is an important marker of apoptosis. Compared with the control group, Bcl-2 was significantly increased in the liriodendrin group, while Cleaved caspase- 3 and Bax were down-regulated (Figure 6). These results indicated that the anti-apoptosis effect of cardiomyocytes was enhanced after treatment with liriodendrin, and the further apoptosis of cardiomyocytes was inhibited. 


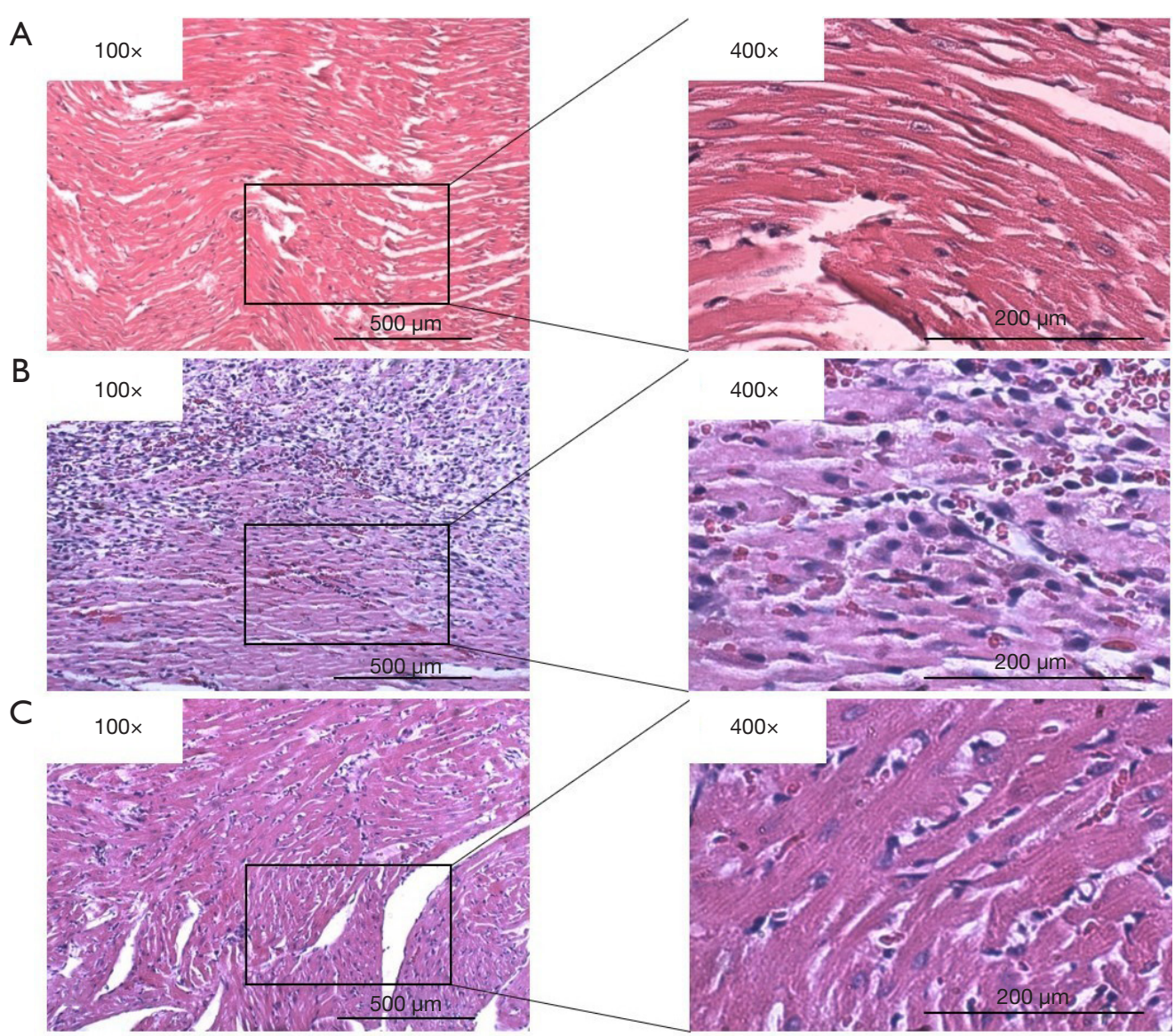

Figure 3 Hearts were stained with HE to observe tissue morphology. (A) The sham group. Liriodendrin alleviated MI damage in rats: (B) collagen deposition was greatest in the control group, myocardial tissue structure disappeared, and many inflammatory cells infiltrated in the damaged region; (C) the least damage was observed in the liriodendrin group. HE, hematoxylin and eosin; MI, myocardial infarction.

\section{Liriodendrin inbibited the transcriptional activity of apoptosis proteins in rats with MI}

To clarify the mechanisms of the anti-apoptosis impact of liriodendrin on MI, mRNA levels of major markers of apoptosis were examined using RT-PCR analysis. As shown in Figure 7, the mRNA levels of Bcl-2 were significantly increased after oral liriodendrin therapy, whereas the levels of Bax and caspase- 3 were reduced compared to the control group $(\mathrm{P}<0.05)$ (Figure 7).

\section{Liriodendrin inbibited the activation of NF- $\mathrm{\kappa B}$ pathways in rats with $M I$}

The anti-inflammatory effects of liriodendrin in rats with MI were subsequently defined by analyzing the activation of
$\mathrm{NF}-\kappa \mathrm{B}$ pathways using western blotting. Increased NF- $\kappa \mathrm{B}$ and phosphorylation of NF- $\mathrm{KB}$ were observed in the control group compared to the sham group. However, reduction

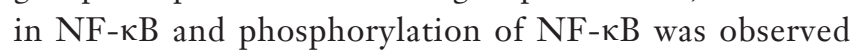
after liriodendrin preprocessing. Moreover, MI caused an obvious phosphorylation of $\mathrm{I} \kappa \mathrm{B} \alpha$, which was in agreement with the NF-kB-binding activity (Figure 8). Liriodendrin was able to prevent the phosphorylation of IкB $\alpha$.

Transcriptional activities of P65 (NF- $\kappa \mathrm{B}), \mathrm{I} \kappa \mathrm{B} \alpha$, and IKK- $\beta$ in the MI area were detected. Liriodendrin inhibited the transcriptional activities of these three proteins compared to the control group $(\mathrm{P}<0.05)$ (Figure 9). IKK- $\beta$, as a key protein in the NF- $\kappa \mathrm{B}$ pathway, promoted phosphorylation of I $\mathrm{KB}$ to activate the NF- $\kappa \mathrm{B}$ pathway. The inhibition of its transcriptional activity indicated that the activation of the $\mathrm{NF}-\kappa \mathrm{B}$ pathway was significantly inhibited by liriodendrin. 

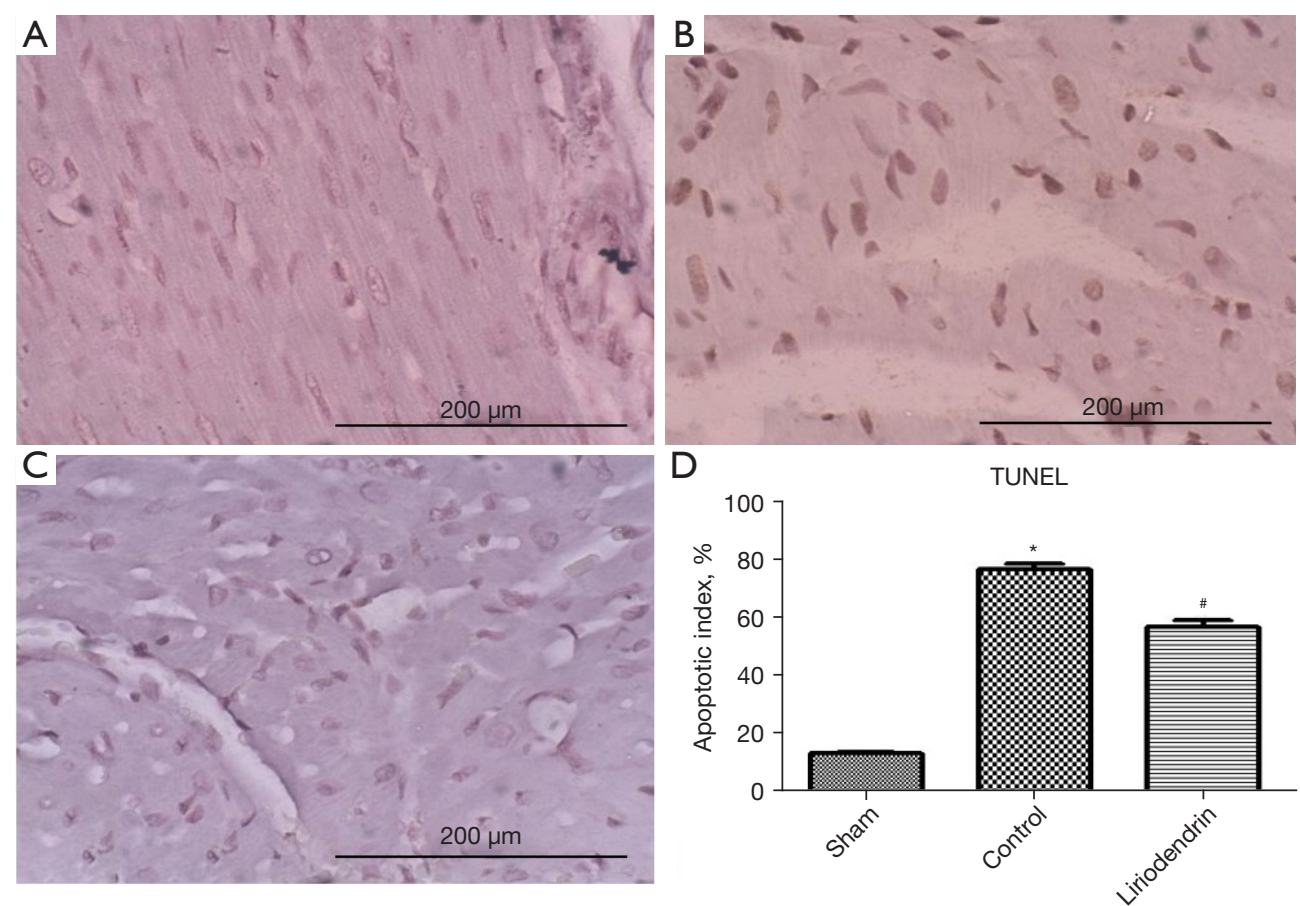

Figure 4 Effect of liriodendrin on anti-apoptosis in myocardium stained with TUNEL. Apoptotic cells are characterized by dark brown nuclei. (A) Sham group; (B) control group; (C) liriodendrin group; and (D) semiquantitative analysis of myocardial tissues by apoptosis index showed that apoptosis was suppressed by liriodendrin and that apoptosis was the most significant in the control group. *: control group $v s$. sham group, $\mathrm{P}<0.05$; " : control group vs. liriodendrin group, $\mathrm{P}<0.05$. TUNEL, transferase-mediated nick end labeling.
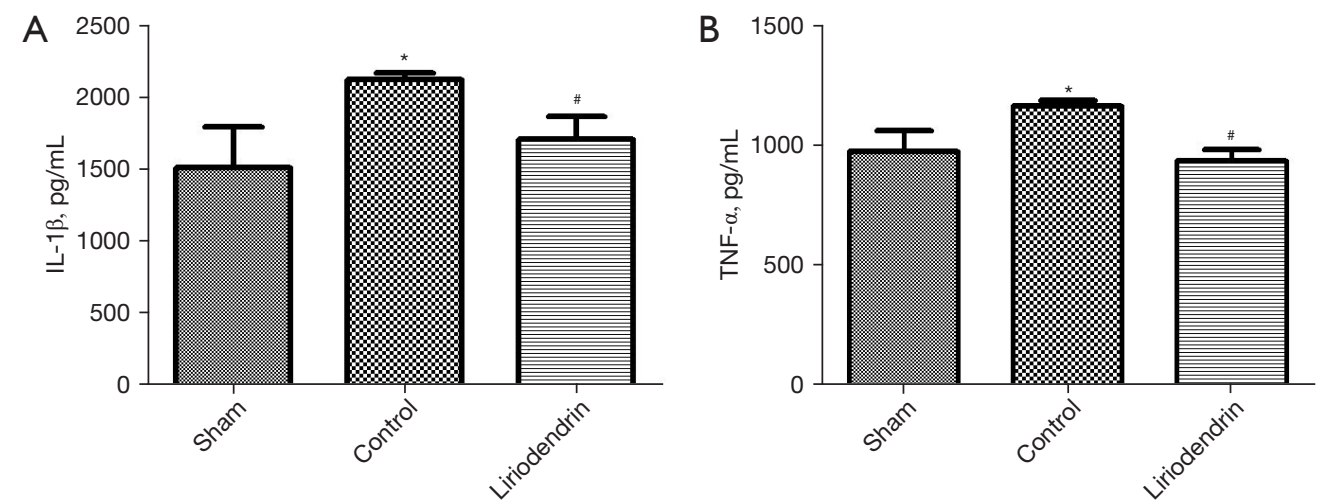

Figure 5 Treatment impact of liriodendrin on concentrations of TNF- $\alpha$ and IL-1 $\beta$ in myocardial tissue. Rat hearts were collected after euthanization. Left ventricular myocardial tissue was homogenized and sonicated. After the centrifuge, the supernatant was obtained to measure $\mathrm{IL}-1 \beta$ (A) and TNF- $\alpha$ (B). Liriodendrin treatment remarkedly inhibited the release of inflammatory factors compared to the control group. *: control group vs. sham group, $\mathrm{P}<0.05$; $^{\#}$ : control group $v$ s. liriodendrin group, $\mathrm{P}<0.05$. IL- $1 \beta$, interleukin- $1 \beta$; TNF- $\alpha$, tumor necrosis factor- $\alpha$.

\section{Liriodendrin inbibited apoptosis, inflammatory cytokine production, and the NF-кB patbway in ischemia-bypoxia stimulated $\mathrm{H} 9 \mathrm{C} 2$ cells}

To further clarify the therapeutic effects of liriodendrin, we defined the anti-inflammatory and anti-apoptosis effects of liriodendrin in $\mathrm{H} 9 \mathrm{C} 2$ cells that had ischemiahypoxia. We pre-treated H9C2 cells with three kinds of concentrations of liriodendrin $24 \mathrm{~h}$ prior to ischemia- 
A
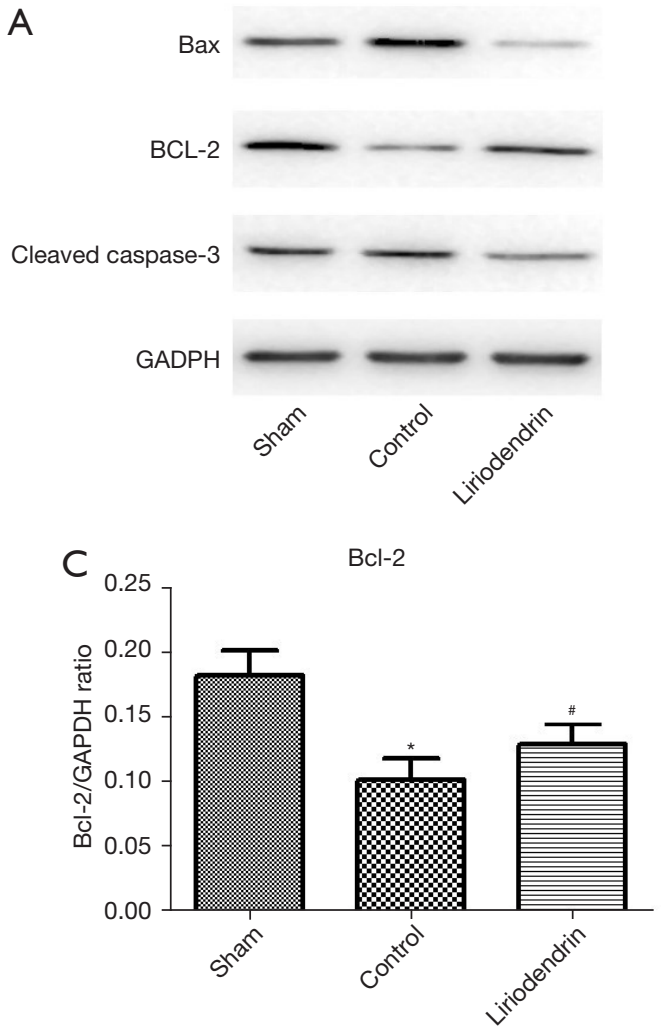

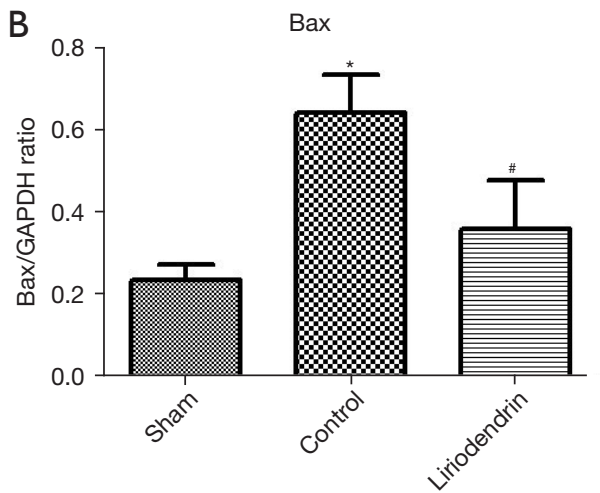

Caspase-3

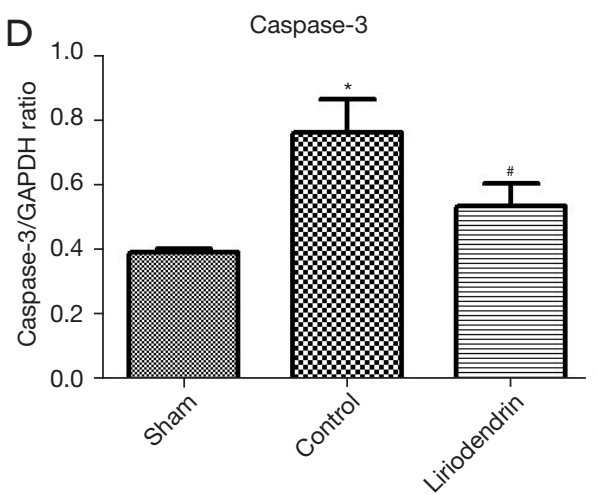

Figure 6 The anti-apoptosis ability of cardiomyocytes in rats with MI was enhanced after liriodendrin treatment. GAPDH was used as a loading control. (A,B) On the contrary, Bax and caspase-3 proteins were reduced in the liriodendrin group compared to the control group; (C) Bcl-2 protein was increased in the liriodendrin group. All results above suggested that apoptosis was inhibited after liriodendrin was administered. *: control group vs. sham group, $\mathrm{P}<0.05$; ": control group vs. liriodendrin group, $\mathrm{P}<0.05$. MI, myocardial infarction; Bcl-2, B-cell lymphoma-2; GAPDH, glyceraldehyde-3-phosphate dehydrogenase.
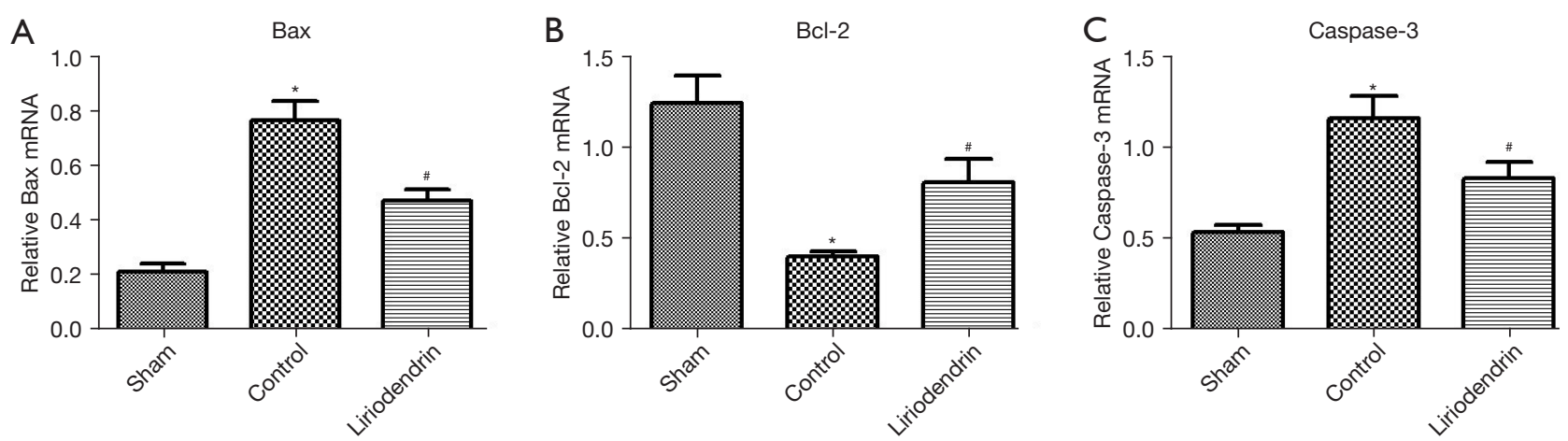

Figure 7 After Liriodendrin treatment, the transcriptional activity of apoptosis was down-regulated in rats with MI. Liriodendrin reduced the mRNA levels of Bax (A), Bcl-2 (B), caspase-3 (C). Real-time PCR assay was performed in duplicate, and GAPDH mRNA was used to normalize the relative amount of mRNA. *: control group $v$ s. sham group, $\mathrm{P}<0.05$; " : control group $v$ s. liriodendrin group, $\mathrm{P}<0.05$. MI, myocardial infarction; Bcl-2, B-cell lymphoma-2; GAPDH, glyceraldehyde-3-phosphate dehydrogenase.

hypoxia exposure. As show in Figure 10, inflammatory factors decreased significantly with increasing liriodendrin concentration, and the most notable change was observed in the $100 \mu \mathrm{M}$ of liriodendrin group. Western blotting analysis showed that expression of Cleaved caspase-3 and Bax proteins were significantly down-regulated, and 

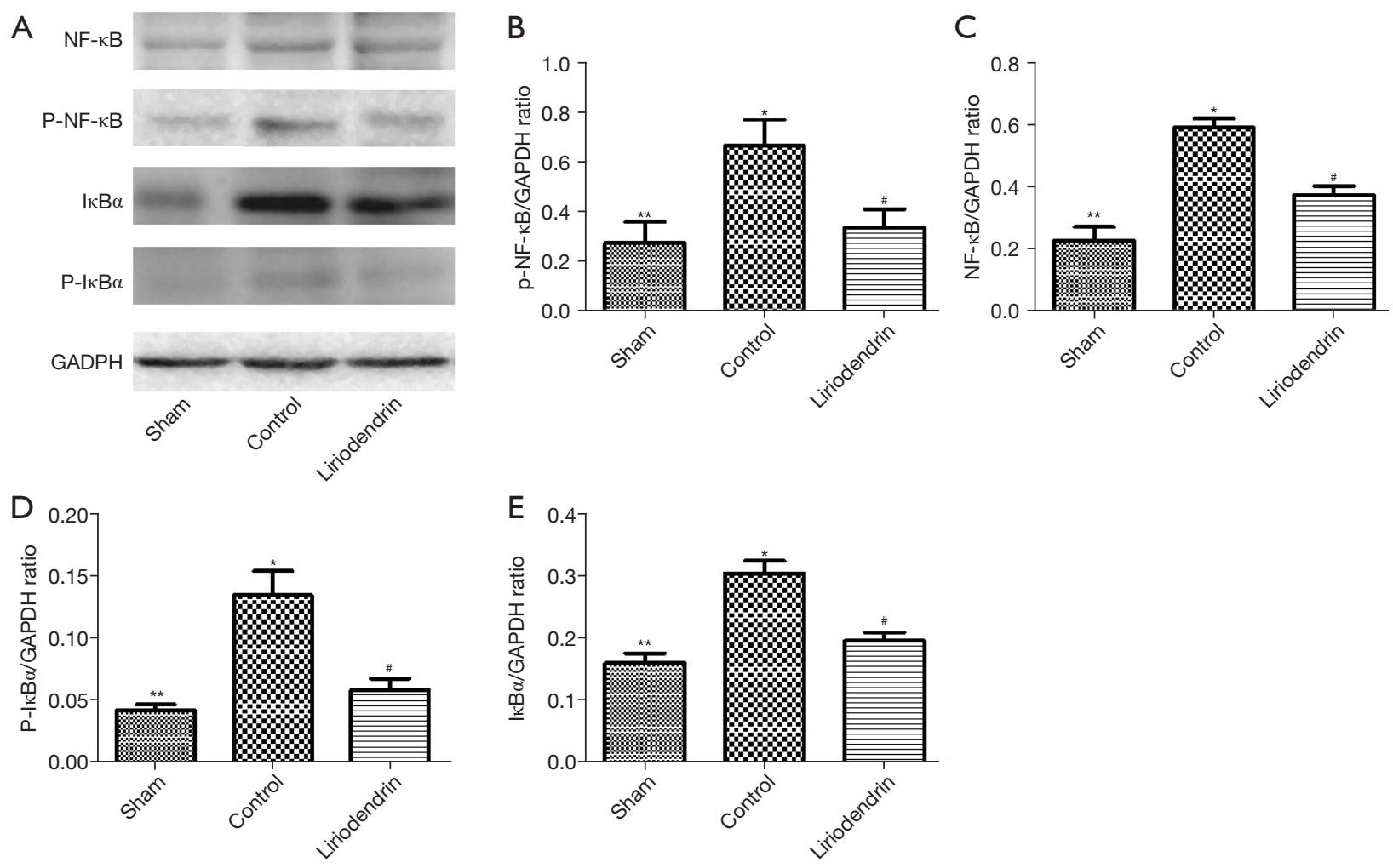

Figure 8 Effect of liriodendrin on activation of NF- $\kappa$ B. (A) Proteins obtained from the myocardium were analyzed by western blot using an enhanced chemiluminescence kit. (B,D) Each band was normalized to that of GAPDH. Liriodendrin inhibited NF- $\kappa \mathrm{B}$ and I $\kappa \mathrm{B} \alpha$ phosphorylation in the myocardium of rats. (C,E) Quantitated density of NF- $\kappa \mathrm{B}$ and $\mathrm{I} \kappa \mathrm{B} \alpha$ in the myocardium. *: control group $v s$. sham group, $\mathrm{P}<0.05$; \#: control group vs. liriodendrin group, $\mathrm{P}<0.05 ;{ }^{* *}$ : liriodendrin group $v$ s. sham group, $\mathrm{P}<0.05$. $\mathrm{NF}-\kappa \mathrm{B}$, nuclear factor kappa-B; GAPDH, glyceraldehyde-3-phosphate dehydrogenase.

expression of Bcl-2 protein was up-regulated (Figure 10). These results indicated that liriodendrin inhibited apoptosis and the secretion of inflammatory factors of $\mathrm{H} 9 \mathrm{C} 2$ cells, and this protective effect was positively correlated with the change of liriodendrin concentration. The NF- $\mathrm{kB}$ pathway can promote apoptosis of cells. The results of western blotting also showed that phosphorylation of NF- $\mathrm{KB}$ and phosphorylation of I $\mathrm{K} \mathrm{B} \alpha$ decreased gradually with the increase of liriodendrin concentration, which was consistent with the trend of apoptosis.

\section{Discussion}

Myocardial infarction has always been considered a disease related to inflammation and apoptosis. Previous studies have shown that inflammation plays an important role in the pathophysiological process of ventricular remodeling after MI (7). Activation of the endogenous immune system causes many inflammatory cells to accumulate in the infarction area, which releases different kinds of chemokines and pro-inflammatory factors and leads to an inflammatory cascade reaction (8). The histopathological results in our study showed that many of inflammatory cells infiltrated in the infarction area in the control group, which was one of the factors affecting ventricular remodeling after MI. In addition, ischemia and hypoxia caused by MI in myocardial tissue resulted in necrosis of myocardial cells. This activated different pathways, including the NF- $\mathrm{BB}$ pathway, which induced apoptosis of myocardial cells and further aggravated ventricular remodeling. These are the main causes of ventricular remodeling after myocardial infarction.

$\mathrm{EF}, \mathrm{ESV}$, and LVIDs reflect cardiac systolic function, while EDV and LVIDd reflect cardiac diastolic function. In this study, liriodendrin significantly lowered LVIDd, LVIDs, ESV, and EDV, which indicated that liriodendrin 

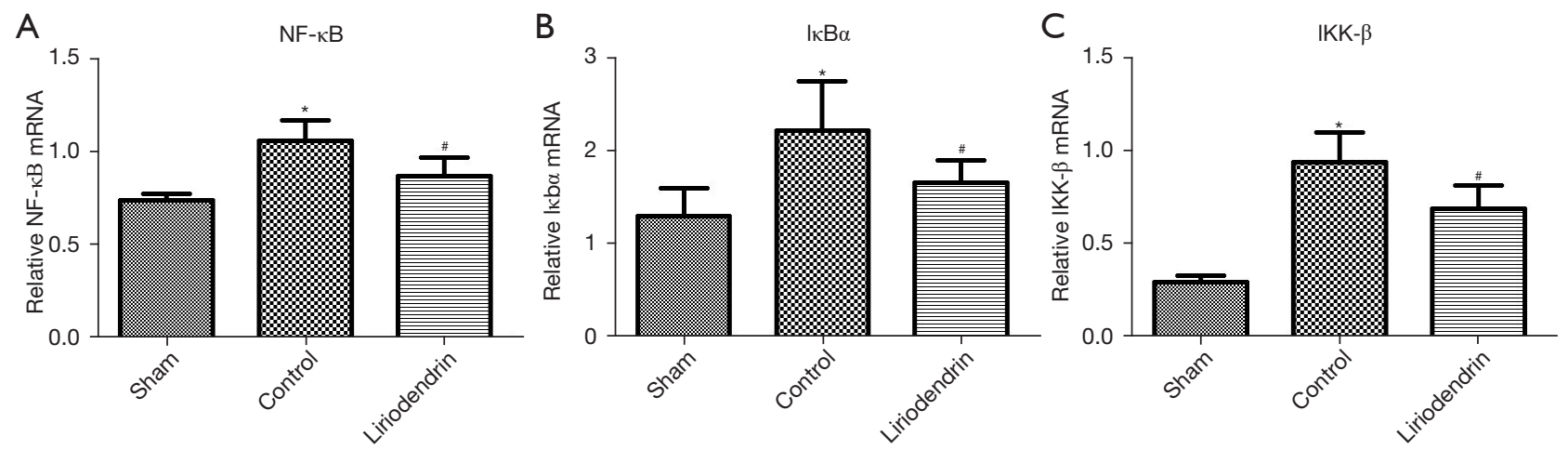

Figure 9 After Liriodendrin treatment, the transcriptional activity of the NF- $\kappa$ B pathway was down-regulated in rats with MI. After RT-


sham group, $\mathrm{P}<0.05$; ": control group vs. liriodendrin group, $\mathrm{P}<0.05$. NF- $\mathrm{B}$, nuclear factor kappa-B; MI, myocardial infarction; RT-PCR, real-time polymerase chain reaction.
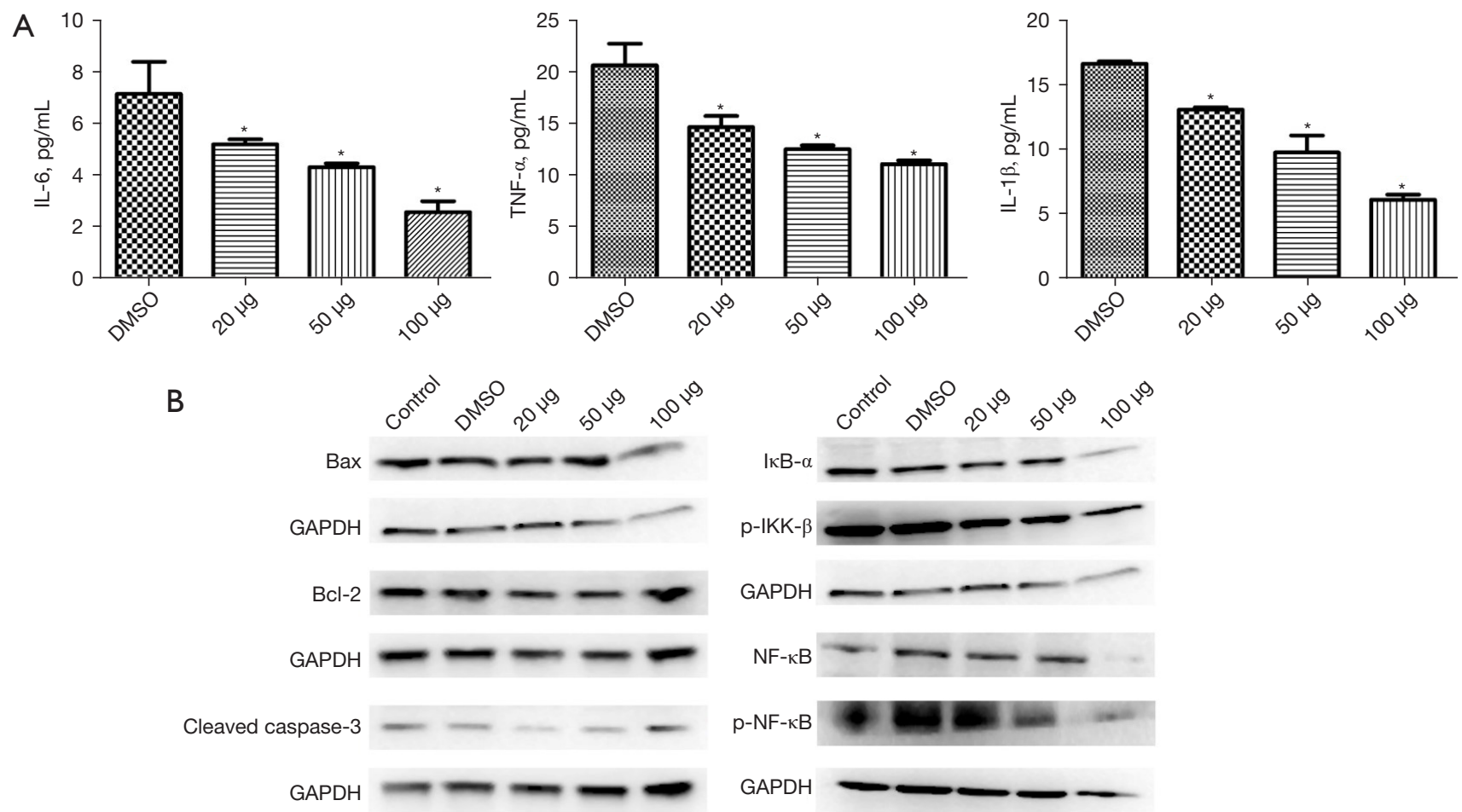

Figure 10 Effects of liriodendrin treatment on upregulated expression of cytokines, TNF- $\alpha$, IL-1 $\beta$, and IL-6 in ischemia-hypoxia stimulated H9C2 cells. (A) Different concentrations of liriodendrin were given $24 \mathrm{~h}$ before ischemia-hypoxia exposure. Cell culture media were harvested $24 \mathrm{~h}$ after the process of ischemia-hypoxia, and the concentrations of TNF- $\alpha$, IL- $1 \beta$, and IL- 6 were measured by ELISA assay. (B) Liriodendrin downregulated the $\mathrm{NF}-\kappa \mathrm{B}$ pathway and apoptosis in $\mathrm{H} 9 \mathrm{C} 2$ cells, and the therapy effect was proportional to the concentration of liriodendrin. ${ }^{*} \mathrm{P}<0.05$, compared with the DMSO group. IL, interleukin; DMSO, dimethyl sulfoxide; ELISA, enzymelinked immunosorbent assay. 
treatment can improve cardiac compliance and cardiac function. HE staining showed that tissue necrosis and inflammatory cell infiltration were observed after MI, which suggested that liriodendrin could significantly inhibit myocardial tissue necrosis, and cardiomyocyte degeneration and inflammatory cell infiltration were improved. These results also demonstrated that liriodendrin may rescue parts of the damaged myocardium, reduce the size of myocardial infarction, and protect myocardial cells in rats with myocardial infarction by inhibiting inflammation.

Several studies have shown that liriodendrin can decrease the production and secretion of inflammatory mediators such as IL- $1 \beta$ and TNF- $\alpha$ in a serum of MI animal models $(3,9,10)$. In this study, cytokines IL-1 $\beta$ and TNF- $\alpha$ in myocardial tissue in the liriodendrin group were lower than those in the control group. The anti-inflammatory effect of liriodendrin was similar to that of the previous study, which indicated that liriodendrin had a suppressive effect on the pulmonary inflammatory response induced by acute lung injury, inhibited the release of inflammatory factors such as IL-6 and TNF- $\alpha$, and inhibited lung damage caused by oxidative stress (4). Our study in vitro confirmed that liriodendrin inhibited the release of inflammatory factors in $\mathrm{H} 9 \mathrm{C} 2$ cells compared to the control group, and the anti-inflammatory effect became more significant with the increase of dosage. These results suggest that liriodendrin can effectively prevent inflammatory cells infiltration and the release of cytokines in MI regions.

Apoptosis is a key pathological process in MI (11). Bax, as a pro-apoptotic protein, can initiate the caspase cascade reaction and activate caspase- 3 to mediate cell death by regulating mitochondrial membrane permeability. caspase- 3 is the most critical pro-apoptotic protein downstream from the caspase cascade reaction (12), and Bcl-2, which has an anti-apoptotic effect, can inhibit the activation of Bax and then inhibit the activation of downstream caspase-3. Furthermore, apoptosis is suppressed. In this study, TUNEL staining showed that the apoptosis index in the liriodendrin group was notably lower than that in the control group. Bax and caspase- 3 proteins were significantly decreased, and Bcl-2 protein was increased in the liriodendrin group. Meanwhile, the transcriptional activities of Bax and caspase- 3 were inhibited. All results above suggested that liriodendrin down-regulated myocardial apoptosis both in vivo and in vitro.

The NF- $\kappa \mathrm{B}$ pathway participates in the transcription and synthesis of inflammatory factors (13-15) and also activates apoptosis (16). Phosphorylated IкB $\alpha$ can promote the release of $\mathrm{NF}-\kappa \mathrm{B}$, which enters the cell nucleus and activates gene expression (17). However, liriodendrin effectively inhibited the phosphorylation of I $\mathrm{KB} \alpha$ and the activation of the NF- $\mathrm{KB}$ pathway both in vivo and in vitro. IKK- $\beta$, as a phosphorylated kinase of IкB $\alpha$, is a key protein involved in the activation of the NF- $\mathrm{KB}$ pathway (17). Its transcriptional activity was inhibited, suggesting that liriodendrin may down-regulate IKK- $\beta$ expression and inhibit the phosphorylation of $\mathrm{I} \kappa \mathrm{B} \alpha$. Moreover, NF- $\kappa \mathrm{B}$ release and nuclear translocation were inhibited, and NF- $\kappa B$ pathway activity was suppressed. Our study also suggested that liriodendrin lowered the expression of inflammatory factors in the tissues and found that liriodendrin inhibits the activity and expression of the NF- $\mathrm{BB}$ pathway. We further used $\mathrm{H} 9 \mathrm{C} 2$ cells to verify in vitro that, with the increase of liriodendrin concentration, this protective effect was positively stronger mainly by means of down-regulating the apoptosis of cells, reducing the secretion of inflammatory factors, and inhibiting the NF- $\kappa B$ pathway.

\section{Conclusions}

Liriodendrin can effectively improve ventricular remodeling and cardiac function after MI in rats. This protective effect may be correlated with the inhibition of the NF$\kappa \mathrm{B}$ pathway, the decrease in inflammatory factors, and the down-regulation of apoptosis. These findings show that liriodendrin could be a new effective agent in order to prevent and treat myocardial injury after MI.

\section{Acknowledgments}

Funding: None.

\section{Footnote}

Reporting Checklist: The authors have completed the ARRIVE reporting checklist. Available at https://jtd. amegroups.com/article/view/10.21037/jtd-21-1998/rc

Data Sharing Statement: Available at https://jtd.amegroups. com/article/view/10.21037/jtd-21-1998/dss

Conflicts of Interest: All authors have completed the ICMJE uniform disclosure form (available at https://jtd.amegroups. com/article/view/10.21037/jtd-21-1998/coif). The authors have no conflicts of interest to declare.

Ethical Statement: The authors are accountable for all 
aspects of the work in ensuring that questions related to the accuracy or integrity of any part of the work are appropriately investigated and resolved. The study was approved by the Animal Ethics Committee of Tianjin Chest Hospital (No. TJCH-2021-007). This work was performed in compliance with the guidelines of the Chinese Ethics Committee for the care and use of animals.

Open Access Statement: This is an Open Access article distributed in accordance with the Creative Commons Attribution-NonCommercial-NoDerivs 4.0 International License (CC BY-NC-ND 4.0), which permits the noncommercial replication and distribution of the article with the strict proviso that no changes or edits are made and the original work is properly cited (including links to both the formal publication through the relevant DOI and the license). See: https://creativecommons.org/licenses/by-nc-nd/4.0/.

\section{References}

1. Yang M, Xiong J, Zou Q, et al. Chrysin attenuates interstitial fibrosis and improves cardiac function in a rat model of acute myocardial infarction. J Mol Histol 2018;49:555-65.

2. Stujanna EN, Murakoshi N, Tajiri K, et al. Rev-erb agonist improves adverse cardiac remodeling and survival in myocardial infarction through an anti-inflammatory mechanism. PLoS One 2017;12:e0189330.

3. Gao S, Li L, Li L, et al. Effects of the combination of tanshinone IIA and puerarin on cardiac function and inflammatory response in myocardial ischemia mice. J Mol Cell Cardiol 2019;137:59-70.

4. Yang L, Li D, Zhuo Y, et al. Protective Role of Liriodendrin in Sepsis-Induced Acute Lung Injury. Inflammation 2016;39:1805-13.

5. Zhang Z, Yang L, Wang B, et al. Protective role of liriodendrin in mice with dextran sulphate sodium-induced ulcerative colitis. Int Immunopharmacol 2017;52:203-10.

6. Labotatory animal-guideline for ethical review of animal welfare. GB/T35892-2018 (2018).

Cite this article as: Li B, Yao BC, Chen QL, Song YQ, Jiang N, Zhao LL, Guo ZG. The protective role and mechanism of liriodendrin in rats with myocardial infarction. J Thorac Dis 2022;14(1):135-146. doi: 10.21037/jtd-21-1998
7. Frangogiannis NG, Smith CW, Entman ML. The inflammatory response in myocardial infarction. Cardiovasc Res 2002;53:31-47.

8. Jiang Q, Yi M, Guo Q, et al. Protective effects of polydatin on lipopolysaccharide-induced acute lung injury through TLR4-MyD88-NF-кB pathway. Int Immunopharmacol 2015;29:370-6.

9. Chi Y, Ma Q, Ding XQ, et al. Research on protective mechanism of ibuprofen in myocardial ischemiareperfusion injury in rats through the PI3K/Akt/ mTOR signaling pathway. Eur Rev Med Pharmacol Sci 2019;23:4465-73.

10. Wang Q, Gong X, Jia J, et al. Protective effect and mechanism of Ribociclib on sepsis induced-acute kidney injury. Zhonghua Wei Zhong Bing Ji Jiu Yi Xue 2020;32:204-9.

11. Abbate A, Narula J. Role of apoptosis in adverse ventricular remodeling. Heart Fail Clin 2012;8:79-86.

12. Hotchkiss RS, Nicholson DW. Apoptosis and caspases regulate death and inflammation in sepsis. Nat Rev Immunol 2006;6:813-22.

13. Zhang X, Du Q, Yang Y, et al. The protective effect of Luteolin on myocardial ischemia/reperfusion (I/R) injury through TLR4/NF-кB/NLRP3 inflammasome pathway. Biomed Pharmacother 2017;91:1042-52.

14. Zhang JJ, Peng K, Zhang J, et al. Dexmedetomidine preconditioning may attenuate myocardial ischemia/ reperfusion injury by down-regulating the HMGB1TLR4-MyD88-NF-кB signaling pathway. PLoS One 2017;12:e0172006.

15. Yuan L, Dai X, Fu H, et al. Vaspin protects rats against myocardial ischemia/reperfusion injury (MIRI) through the TLR4/NF- $\mathrm{BB}$ signaling pathway. Eur J Pharmacol 2018;835:132-9.

16. Nogami H, Urist MR. The classic : a morphogenetic matrix for differentiation of cartilage in tissue culture. Clin Orthop Relat Res 2009;467:3063-7.

17. Prescott JA, Mitchell JP, Cook SJ. Inhibitory feedback control of NF- $\kappa \mathrm{B}$ signalling in health and disease. Biochem J 2021;478:2619-64. 